\title{
Resist Thickness Dependence of Latent Images in Chemically Amplified Resists Used for Electron Beam Lithography
}

\author{
Takahiro Kozawa ${ }^{1 *}$ and Takao Tamura ${ }^{2}$ \\ ${ }^{1}$ The Institute of Scientific and Industrial Research, Osaka University, \\ 8-1 Mihogaoka, Ibaraki, Osaka 567-0047, Japan \\ ${ }^{2}$ NuFlare Technology, Inc., 8-1 Shinsugita, Isogo, Yokohama, 235-8522, Japan \\ "kozawa@sanken.osaka-u.ac.jp
}

\begin{abstract}
Transistors have been miniaturized to increase their integration. With miniaturization, the thickness of the patterning material, called a resist, has been decreased to prevent them from collapsing. In this study, the resist film thickness dependence of the latent images of chemically amplified electron beam resists was investigated using the simulation on the basis of their sensitization and reaction mechanisms. The decompositions of sensitizers (photoacid generators) were significantly affected by the dynamics of low-energy electrons at the interfaces of resist films. The subsequent deprotection was also affected by the initial acid distribution. Simulation results indicated that such interfacial effects basically increased with the decrease of resist film thickness.
\end{abstract}

Keywords: EB lithography, Chemically amplified resist, Interface effect, Low-energy electron

\section{Introduction}

The electron beam (EB) lithography is an indispensable technology for the information-oriented society. It has been used for the fabrication of photomasks, extreme ultraviolet (EUV) masks, and nanoimprint molds for the high-volume production of semiconductor devices. The requirement for photomasks becomes severe with the miniaturization of electronic devices. When the critical dimension was decreased, the resist patterns tend to collapse due to the surface tension of rinse liquid (pure water) [1-3]. The collapse prevention measures such as freeze drying process, supercritical drying, and dry development rinse process have been investigated [4-8]. They are, however, still inadequate or impose additional costs. Therefore, the reduction of resist film thickness is required for the high-resolution patterning. However, the quality of resist patterns is degraded with the decrease of the initial resist film thickness for the sub-100 $\mathrm{nm}$ thickness range, even if the pattern collapse is avoided [9-12].

A highly sensitive resist, called a chemically amplified resist, has been used for the fabrication of photomasks [13]. The sensitization mechanism has been reported as follows. The resist molecules are ionized and secondary electrons are emitted upon by the irradiation of electron beam. The counteranions of acids are generated through the dissociative electron attachment to acid generators after the secondary electrons are thermalized $[14,15]$. The protons of acids are mainly generated through the deprotonation of polymer radical cations [16,17]. The generated acids catalyze the deprotection of polymer molecules during the postexposure baking (PEB). The deprotection induces the polarity change of polymer molecules, which leads to the solubility change of resist film in the tetramethylammonium hydroxide (TMAH) aqueous developer. The low-energy electrons play an essential role in the sensitization of 
chemically amplified EB resists.

The resist film is roughly divided into three layers (near-surface, bulk, and near-substrate layers). With the decrease of initial resist film thickness, the volume of bulk layer decreases and the effects of near-surface and near-substrate layers on the resist performance increase. For the near-surface layer, the segregation of low molecular weight components was investigated [18-23]. Such surface segregation was reported to lead to the fast deprotection at the near-surface layer [18]. The segregation at the near-substrate layer was weaker than that at the near-surface layer [19-23].

The film thickness dependence of glass transition temperature $T_{g}$, which reflects the strength of polymer-substrate interaction, has been investigated [24-27]. When the polymer-substrate interaction is strong, the mobility of acids decreases. The dynamics of low-energy electrons at the interface has been also reported to affect the acid distribution in the depth direction for chemically amplified EUV resists [28]. In this study, the resist film thickness dependence of the latent images of chemically amplified EB resists was investigated, assuming eight patterns for the low-energy electron dynamics at the interfaces. The latent images of $100 \mathrm{~nm}$ line-and-space patterns were calculated for the initial resist film thickness of 20 and 70 $\mathrm{nm}$ on the basis of the sensitization and reaction mechanisms of chemically amplified EB resists [29]. The effect of low-energy electron dynamics at the interfaces on the latent image formation is discussed.

\section{Simulation model and method}

The chemically amplified resist assumed consisted of the triphenylsulfonium nonaflate (TPS-nf) as an acid generator, the trioctylamine (TOA) as a quencher, and a partially protected poly(4-hydroxystyrene) (PHS). The TPS-nf concentration was 0.257 $/ \mathrm{nm}^{3}$. The TOA concentration was $0.128 / \mathrm{nm}^{3}$. A tert-butoxycarbonyl group was assumed as a protecting group. The protected ratio was $31.2 \%$.

The latent images of line-and-space patterns were calculated on the basis of the sensitization and reaction mechanisms of chemically amplified EB resists. The
Table I. Parameters used in the simulation.

\begin{tabular}{ll} 
Acceleration voltage of electron beam $(\mathrm{kV})$ & 125 \\
Beam blur $\left(\sigma_{\mathrm{b}}\right)(\mathrm{nm})$ & 2 \\
Backscattering coefficient[38] & 0.31 \\
Half-pitch $(\mathrm{nm})$ & 100 \\
Initial resist film thickness $(\mathrm{nm})$ & 20,70 \\
Stopping power $(\mathrm{eV} / \mathrm{nm})$ & 0.418 \\
Resist film density $\left(\mathrm{g} / \mathrm{cm}^{3}\right)[21]$ & 1.2 \\
Thermalization distance $(\mathrm{nm})[30]$ & 3.2 \\
Acid generator concentration $\left(/ \mathrm{nm}^{3}\right)$ & 0.257 \\
Quencher concentration $\left(/ \mathrm{nm}^{3}\right)$ & 0.128 \\
Reaction radius of acid generator $(\mathrm{nm})[30,31]$ & 0.70 \\
Effective reaction radius for neutralization & 0.5 \\
(nm) & \\
Effective reaction radius for deprotection & 0.1 \\
(nm) & \\
Protection ratio $(\%)$ & 31.2 \\
Deprotonation efficiency of proton source[39] & 1.0 \\
Deprotonation efficiency of nonproton & 0.59 \\
source[32] & \\
Diffusion constant of acids (nm $\left.{ }^{2} / \mathrm{s}\right)$ & 1.0 \\
PEB time $(\mathrm{s})$ & 600 \\
PEB temperature $\left({ }^{\circ} \mathrm{C}\right)$ & 130 \\
\hline
\end{tabular}

acceleration voltage of the electron beam was $125 \mathrm{kV}$. The beam blur was approximated using a Gaussian function. The beam blur $\left(1 \sigma_{\mathrm{b}}\right)$ was set to $2.0 \mathrm{~nm}$. The pitch was 200 $\mathrm{nm}$ and the calculated area was $200 \times 150$ $\mathrm{nm}^{2}$. The thickness of resist films was 20 and $70 \mathrm{~nm}$. The reaction radius of TPS-nf with thermalized electrons were assumed to be the same as that of triphenylsulfonium triflate (TPS-tf) [30,31]. The thermalization distance of secondary electrons in PHS has been reported to be $3.2 \mathrm{~nm}$.[30] The deprotonation efficiency of tert-butoxycarbonyl protected unit radical cations was set to 0.59 [32]. The trajectories of secondary electrons and the reaction of thermalized electrons with acid generators were calculated by a Monte Carlo method. The low-energy (<ionization potential) electron dynamics at the surface (the interface between vacuum and a resist layer) was categorized into two patterns. The low-energy electron dynamics at the interface between a resist layer and an underlayer (UL) was categorized into four patterns, as later explained. The other details of the calculation procedure have been reported elsewhere [33].

Using the acid distribution after the preneutralization $[34,35]$ as the initial condition, the catalytic chain deprotection during PEB was also calculated by solving the reaction-diffusion equations. The details 
Low-energy electron dynamics at the interfaces
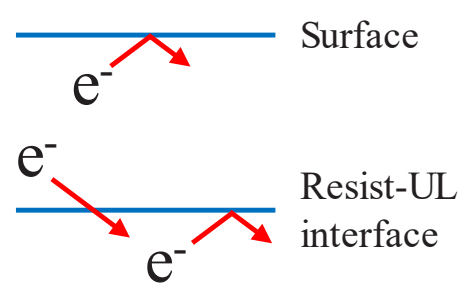

Initial acid distribution before PEB

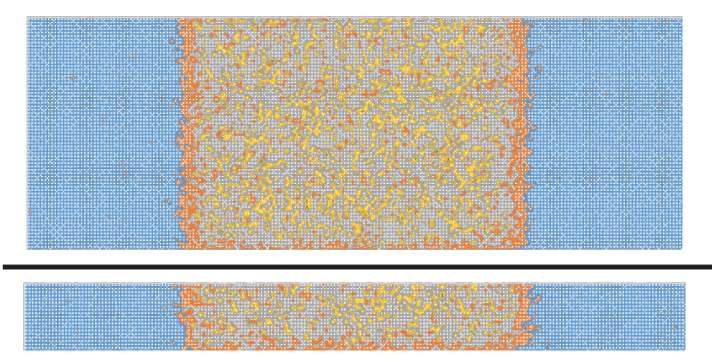

Protected unit distribution after PEB

$0.00-0.05 \square 0.05-0.10 \square 0.10-0.15$

$\square 0.15-0.20 \square 0.20-0.25$

(molecules) $/ \mathrm{nm}^{3}$

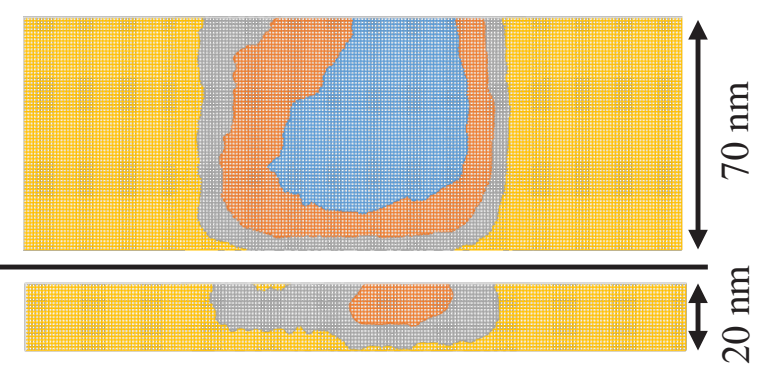

$0.0-0.5 \square 0.5-1.0 \square 1.0-1.5 \square 1.5-2.0$

(molecules) $/ \mathrm{nm}^{3}$

$200 \mathrm{~nm}$

Fig. 1. Initial resist film thickness dependence of initial acid distribution before PEB and protected unit distribution after PEB, obtained by the simulation. The features were line-and-space patterns with $100 \mathrm{~nm}$ half-pitch. The film thicknesses were 20 and $70 \mathrm{~nm}$. The boundary conditions were reflection (from resist layer to vacuum), transmission (from resist to UL), and reflection (from UL to resist). The figures for $20 \mathrm{~nm}$ film thickness were reprinted from Ref. 12 .

of the calculation procedure have been reported elsewhere [36]. The effective reaction radius for neutralization was set to $0.5 \mathrm{~nm}$. The effective reaction radius for deprotection was assumed to be $0.1 \mathrm{~nm}$ [37]. The periodic boundary condition was applied in the horizontal direction. The reflective boundary condition was applied in the vertical direction. The backscattering coefficient was assumed to be 0.31 [38]. The parameters used in the simulation are summarized in Table I [21,30-32,38,39]. The validity of the simulation method has been examined by comparison with the experimental results obtained by using EBM-9000 of NuFlare [37]. Other details of the reaction mechanisms have been reported elsewhere [29].

\section{Results and discussion}

The resist film thickness dependence of the pattern formation of chemically amplified EB resists was investigated using $\mathrm{Si}$ wafers ( $\mathrm{p}$ type, electrical resistance of $\leqq 0.02 \Omega \mathrm{cm}$ ) in our previous study [12]. Using an EB writer $(125 \mathrm{keV})$, the line-and-space patterns with $100 \mathrm{~nm}$ half-pitch were fabricated. The line width roughness (LWR) of line-and-space patterns was analyzed. The dissolution kinetics of resist films was also analyzed using a quartz crystal microbalance (QCM) method. Figure 1 shows the initial resist film thickness dependence of initial acid distribution before $\mathrm{PEB}$ and protected unit distribution after PEB, obtained by the simulation on the basis of the sensitization and reaction mechanisms of chemically amplified EB resists. The simulated latent images well explained the resist film thickness dependence of LWR and dissolution kinetics. In the simulation, the low-energy electrons were assumed to be able to move from the resist layer to UL and to be unable to move from UL to the resist layer because both $\mathrm{Au}$ and $\mathrm{Si}$ wafer used are electrically conductive. For the resist surface, the low-energy electrons were assumed to be unable to escape to the vacuum from the comparison with the crosssectional SEM images of line-and-space patterns. Thus, the boundary conditions for the simulation were set to be the reflection (from resist layer to vacuum), the transmission (from resist to UL), and the reflection (from UL to resist). Under such conditions, the acid concentration 
Low-energy electron

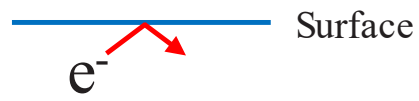

dynamics at the interfaces

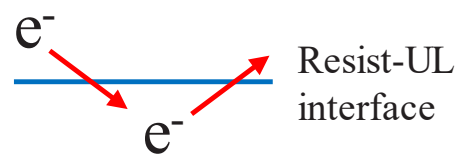

Initial acid distribution before PEB

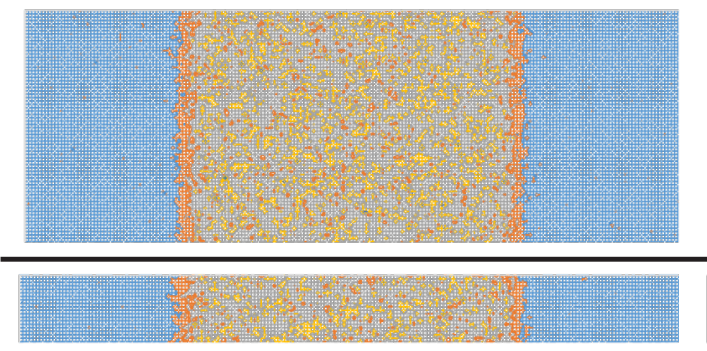

Protected unit distribution after PEB

$\square 0.00-0.05 \square 0.05-0.10 \square 0.10-0.15$

$\square 0.15-0.20 \square 0.20-0.25$

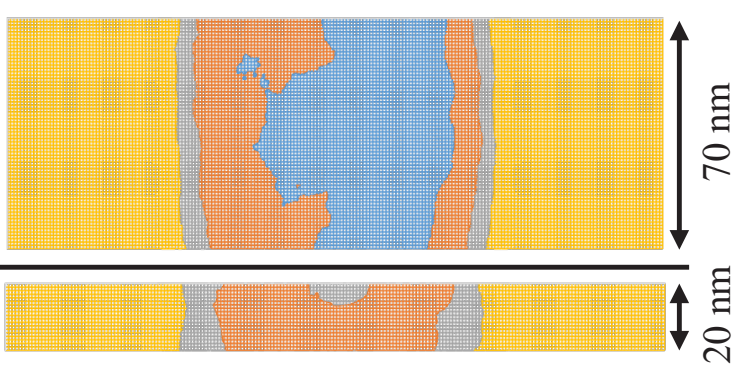

$\square 0.0-0.5 \square 0.5-1.0 \square 1.0-1.5 \square 1.5-2.0$

(molecules) $/ \mathrm{nm}^{3}$

(molecules) $/ \mathrm{nm}^{3}$

Fig. 2. Initial resist film thickness dependence of initial acid distribution before PEB and protected unit distribution after PEB, obtained by the simulation. The boundary conditions were reflection (from resist layer to vacuum), transmission (from resist to UL), and transmission (from UL to resist).

decreased near UL because of the loss of low-energy electrons. There was no difference between the acid distributions near UL for the resists with 20 and $70 \mathrm{~nm}$ film thickness. The acids diffuse in the resist matrix during PEB. The amount of acids supplied from the bulk layer decreased with decreasing the resist film thickness. Consequently, the protected unit concentration near UL for the resist with $20 \mathrm{~nm}$ film thickness became higher than that for the resist with $70 \mathrm{~nm}$ film thickness [12].

Figure 2 shows the initial resist film thickness dependence of initial acid distribution before PEB and protected unit distribution after PEB, obtained by the simulation. The boundary conditions were set to be the reflection (from resist layer to vacuum), the transmission (from resist to UL), and the transmission (from UL to resist). The difference from the case shown in Fig. 1 is the low-energy electron dynamics from UL to the resist layer. The initial acid concentration near UL increased owing to the supply of low-energy electrons from UL. The protected unit concentration near UL became lower than those shown in Fig. 1 because of the increased acids. However, the dependence of protected unit concentration near UL on the initial resist film thickness was still observed.
The reflection boundary condition was applied to both interfaces. The initial acid distribution before PEB and the protected unit distribution after PEB are shown in Fig. 3 for the initial resist film thicknesses of 20 and 70 $\mathrm{nm}$. In this study, the material properties of UL associated with the secondary electron generation and electron deceleration were assumed to be the same as those of the resists. In addition, the penetrating power of $125 \mathrm{keV}$ electron is significantly high. Therefore, the simulation results should be the same as those shown in Fig. 2. The observed difference in the protected unit distribution (Figs. 2 and 3 ) is a calculation error originated from the stochasticity of Monte Carlo method applied to the calculation of acid distribution. The dependence of protected unit concentration near UL on the initial resist film thickness was decreased, unlike the case shown in Fig. 2. With the same reason, the thickness dependence shown in Fig. 3 should be the same as the case shown in Fig. 2. This discrepancy is considered to be caused because the stochasticity strongly affected the protected unit distribution with the decrease of resist film thickness. That is, the number of acids in the target feature decreased with the decrease of resist film thickness. The decrease in the number of acids led to the increase in the stochasticity. 
Low-energy electron dynamics at the interfaces
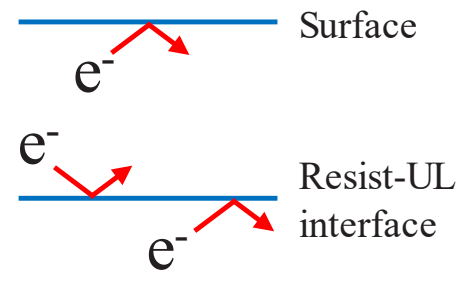

Initial acid distribution before PEB

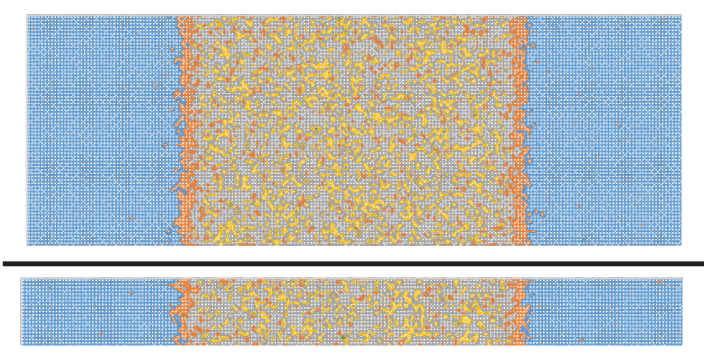

$\square 0.00-0.05 \square 0.05-0.10 \square 0.10-0.15$

$\square 0.15-0.20 \square 0.20-0.25 \square 0.25-0.30$

Protected unit distribution after PEB

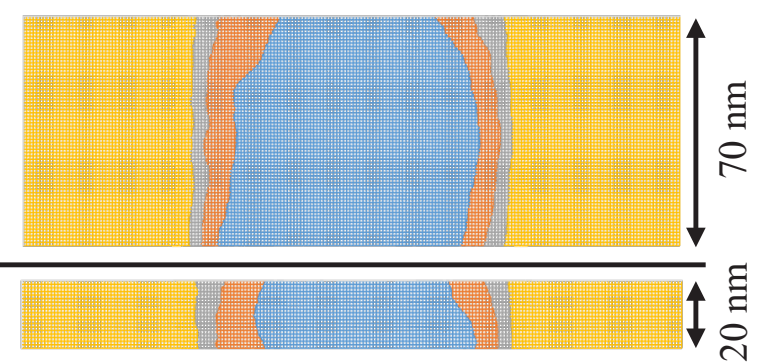

$\square 0.0-0.5 \square 0.5-1.0 \square 1.0-1.5 \square 1.5-2.0$

(molecules) $/ \mathrm{nm}^{3}$

(molecules) $/ \mathrm{nm}^{3}$

Fig. 3. Initial resist film thickness dependence of initial acid distribution before PEB and protected unit distribution after PEB, obtained by the simulation. The boundary conditions were reflection (from resist layer to vacuum), reflection (from resist to UL), and reflection (from UL to resist).

Low-energy electron dynamics at the interfaces
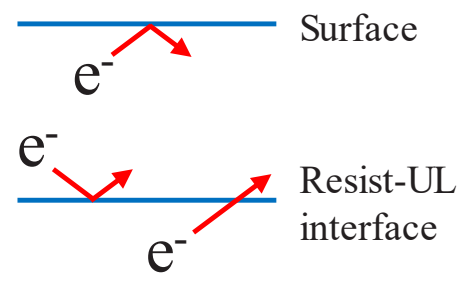

Initial acid distribution before PEB

Protected unit distribution after PEB
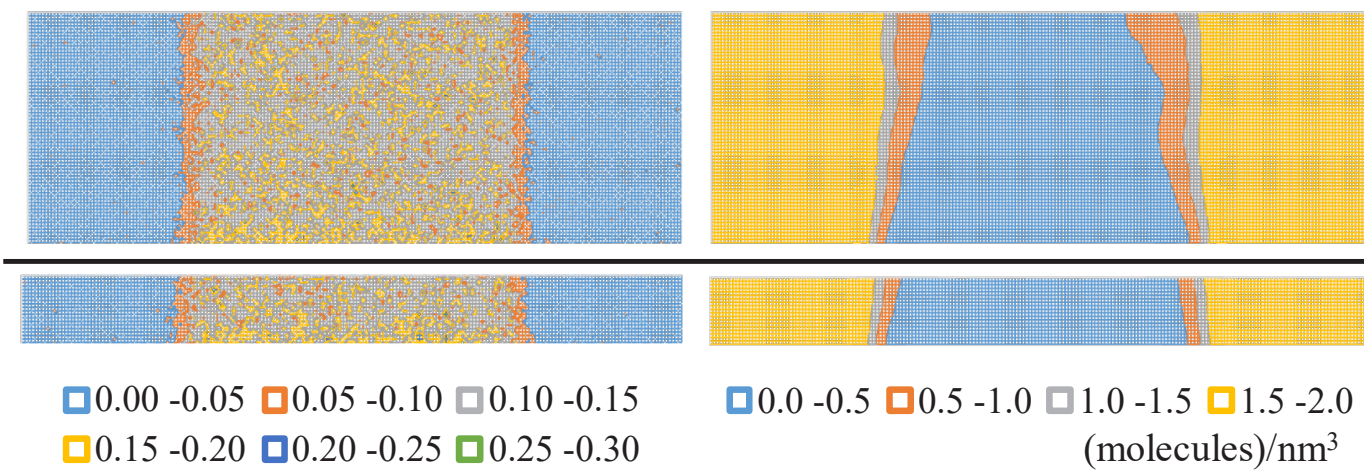

(molecules) $/ \mathrm{nm}^{3}$

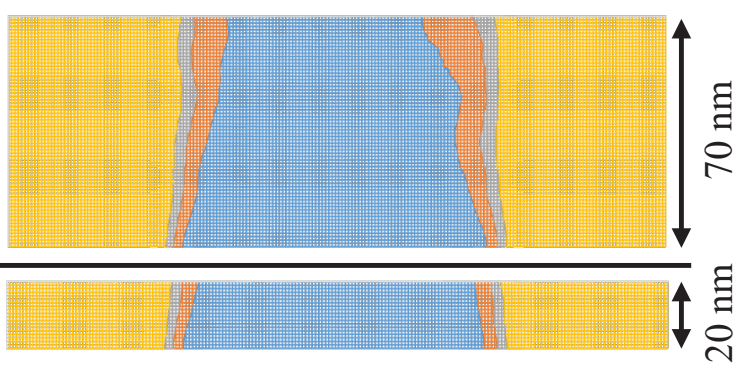

Fig. 4. Initial resist film thickness dependence of initial acid distribution before PEB and protected unit distribution after PEB, obtained by the simulation. The boundary conditions were reflection (from resist layer to vacuum), reflection (from resist to UL), and transmission (from UL to resist).

Figure 4 shows the initial resist film thickness dependence of initial acid distribution before PEB and protected unit distribution after PEB, calculated with the boundary condition that the low-energy electrons can move from UL to the resist layer. The reflection was assumed for the other boundary conditions. The acid concentration near UL increased owing to the influx of low-energy electrons from UL. Consequently, the pattern shape became an inverse tapered shape. The dependence of protected unit concentration near UL on the initial resist film thickness was not observed. 
Low-energy electron

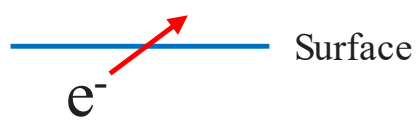

dynamics at the interfaces

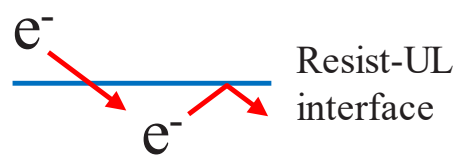

Initial acid distribution before PEB

Protected unit distribution after PEB

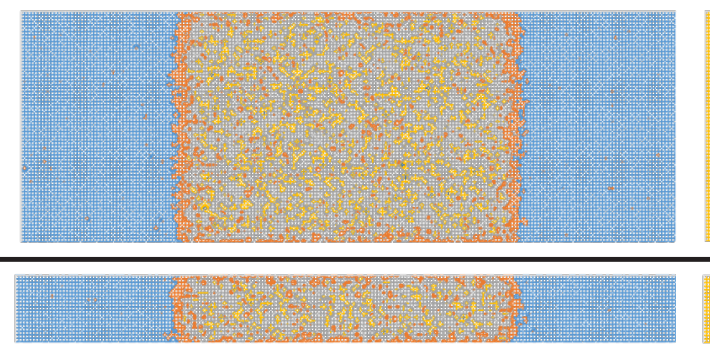

$$
\begin{gathered}
\square 0.00-0.05 \square 0.05-0.10 \square 0.10-0.15 \quad \square 0.0-0.5 \square 0.5-1.0 \square 1.0-1.5 \square 1.5-2.0 \\
\begin{aligned}
\square & \square .15-0.20 \square 0.20-0.25
\end{aligned} \\
\text { (molecules) } / \mathrm{nm}^{3}
\end{gathered}
$$

Fig. 5. Initial resist film thickness dependence of initial acid distribution before PEB and protected unit distribution after PEB, obtained by the simulation. The boundary conditions were transmission (from resist layer to vacuum), transmission (from resist to UL), and reflection (from UL to resist).

Low-energy electron dynamics at the interfaces
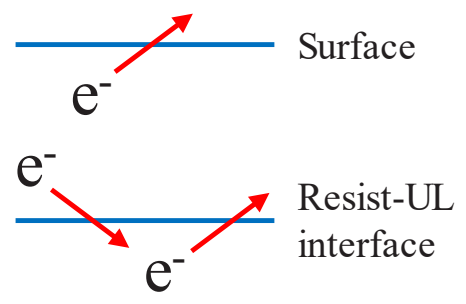

Initial acid distribution before PEB

Protected unit distribution after PEB
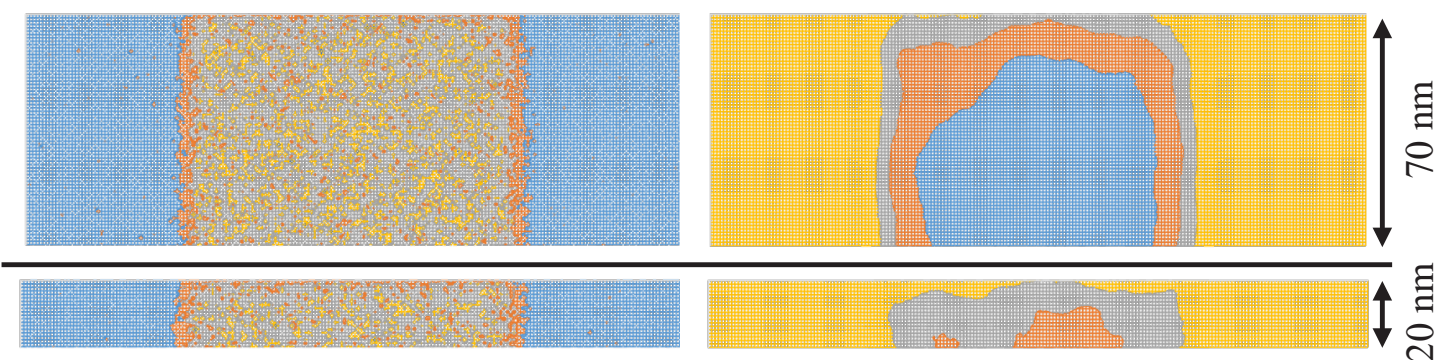

$\square 0.00-0.05 \square 0.05-0.10 \square 0.10-0.15$

$\square 0.15-0.20 \square 0.20-0.25$

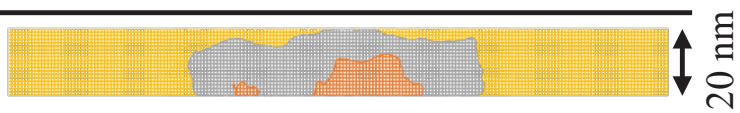

(molecules) $/ \mathrm{nm}^{3}$

$\square 0.0-0.5 \square 0.5-1.0 \square 1.0-1.5 \square 1.5-2.0$

(molecules) $/ \mathrm{nm}^{3}$

Fig. 6. Initial resist film thickness dependence of initial acid distribution before PEB and protected unit distribution after PEB, obtained by the simulation. The boundary conditions were transmission (from resist layer to vacuum), transmission (from resist to UL), and transmission (from UL to resist).

Thus far, the effect of interface between resist film and UL on the initial resist film thickness dependence was discussed. Next, the effect of surface condition is discussed. The boundary conditions of the surfaces were reflection for the cases shown in Figs. 1-4.
Changing the boundary condition of the surface from reflection to transmission, the similar calculation was carried out for all the cases. Figs. 5, 6, 7, and 8 correspond to Figs. $1,2,3$, and 4 , respectively. The protected unit concentration near the surface decreased 
Low-energy electron

dynamics at the interfaces
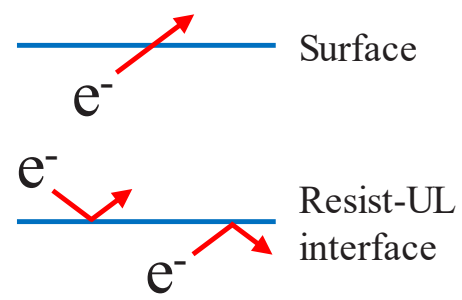

Initial acid distribution before PEB

Protected unit distribution after PEB
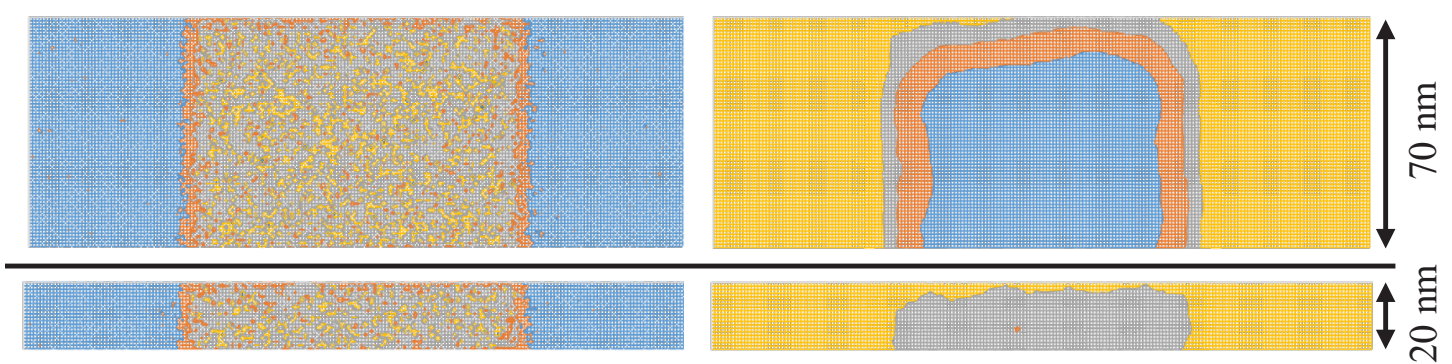

$\square 0.00-0.05 \square 0.05-0.10 \square 0.10-0.15$

$\square 0.15-0.20 \square 0.20-0.25$

$\square 0.0-0.5 \square 0.5-1.0 \square 1.0-1.5 \square 1.5-2.0$

(molecules) $/ \mathrm{nm}^{3}$

(molecules) $/ \mathrm{nm}^{3}$

Fig. 7. Initial resist film thickness dependence of initial acid distribution before PEB and protected unit distribution after PEB, obtained by the simulation. The boundary conditions were transmission (from resist layer to vacuum), reflection (from resist to UL), and reflection (from UL to resist).

Low-energy electron dynamics at the interfaces
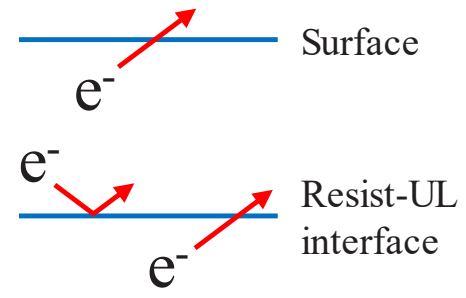

Initial acid distribution before PEB

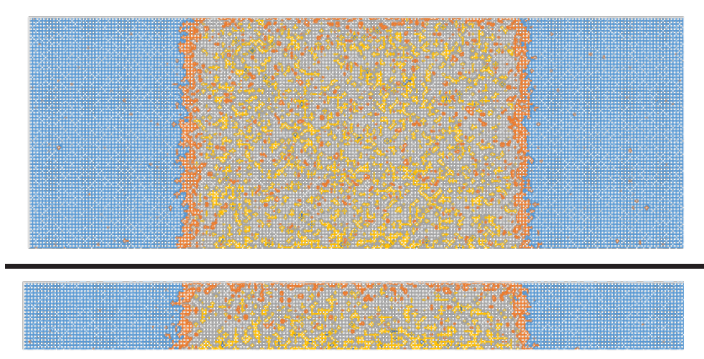

$\square 0.00-0.05 \square 0.05-0.10 \square 0.10-0.15$

$\square 0.15-0.20 \square 0.20-0.25$

Protected unit distribution after PEB

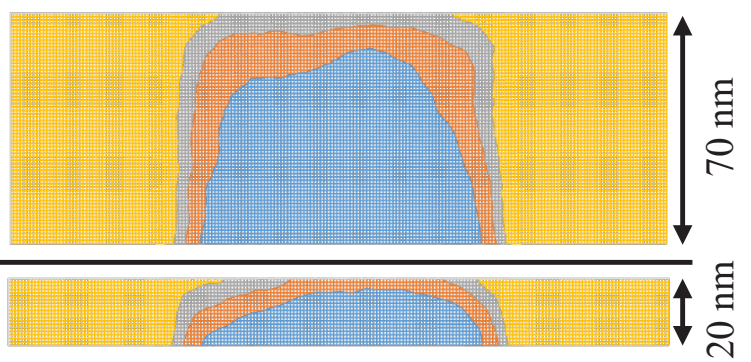

$\square 0.0-0.5 \square 0.5-1.0 \square 1.0-1.5 \square 1.5-2.0$

\section{(molecules) $/ \mathrm{nm}^{3}$}

Fig. 8. Initial resist film thickness dependence of initial acid distribution before PEB and protected unit distribution after PEB, obtained by the simulation. The boundary conditions were transmission (from resist layer to vacuum), reflection (from resist to UL), and transmission (from UL to resist).

owing to the loss of acids caused by the escape of low-energy electrons from the surface. With the reduction of resist film thickness, this effect increased with the same reason discussed previously except for the case shown in Fig. 8. The situation is complicated for the case shown in Fig. 8, because the interfaces at the top and bottom of resist layer have opposite effects. Both effects increase by decreasing the resist film thickness. Consequently, the effects of interfaces seemed to decrease with the 
decrease of initial resist film thickness. The discussion about the comparison between Figs. 2 and 3 described above can also be applied to Figs. 6 and 7. The simulation results in Fig. 6 should be approximately the same as those in Fig. 7. The discrepancy was caused by the stochasticity of Monte Carlo method applied to the calculation of acid distribution. The same discussion can be applied to the real cases.

\section{Conclusion}

The resist film thickness dependence of the latent images of chemically amplified EB resists was investigated using the simulation on the basis of their sensitization and reaction mechanisms. The low-energy electron dynamics at the surface (the interface between vacuum and a resist layer) was categorized into two patterns. The low-energy electron dynamics at the interface between a resist layer and UL was categorized into four patterns. The decomposition of sensitizers (photoacid generators) were significantly affected by the dynamic of low-energy electrons at the interfaces of resist films. The subsequent deprotection was also affected by the initial acid distribution. Such interfacial effects basically increased with the decrease of resist film thickness. These results suggest that the resist formula should be optimized in accordance with the conditions of interfaces when the resist film thickness is reduced.

\section{References}

1. T. Tanaka, M. Morigami, and N. Atoda, Jpn. J. Appl. Phys., 32 (1993) 6059.

2. S. F. Chini and A. Amirfazli, Langmuir, 26 (2010) 13707.

3. A. Kawai and K. Suzuki, Jpn. J. Appl. Phys., 45 (2006) 5429.

4. H. Namatsu, J. Photopolym. Sci. Technol., 15 (2002) 381

5. T. Tanaka, M. Morigami, H. Oizumi, and T. Ogawa, Jpn. J. Appl. Phys., 32 (1993) 5813.

6. K. Shin and G. Lee, J. Photopolym. Sci. Technol., 16 (2003) 363.

7. R. Sakamoto, Y. Sakaida, and B. -C. Ho, Proc. SPIE, 8682 (2013) 868205.

8. M. Harumoto, T. Motono, A. F. Santos, C. Mori, Y. Tanaka, H. Stokes, M. Asai, J. Santillan, T. Itani, and T. Kozawa, Jpn. J. Appl. Phys., 60 (2021) SCCA03.
9. T. Yamaguchi, K. Yamazaki, M. Nagase, and H. Namatsu, Jpn. J. Appl. Phys., 42 (2003) 3755.

10. J. H. Kim, N. Choi, Y. - H. Kim, and T. - S. Kim, Proc. SPIE, 6153 (2006) 615337.

11. L. Singh, I. Matthew, A. Pawloski, and A. Minvielle, Proc. SPIE, $\mathbf{6 1 5 3}$ (2006) $61530 \mathrm{~W}$.

12. N. Maeda, A. Konda, K. Okamoto, T. Kozawa, and T. Tamura, Jpn. J. Appl. Phys., 59 (2020) 086501.

13. H. Ito and C. G. Willson, Polym. Eng. Sci., 23 (1983) 1012.

14. T. Kozawa, Y. Yoshida, M. Uesaka, and S Tagawa, Jpn. J. Appl. Phys., 31 (1992) 4301

15. K. Natsuda, T. Kozawa, K. Okamoto, A Saeki, and S. Tagawa, Jpn. J. Appl. Phys., 48 (2009) 06FC05.

16. A. Nakano, T. Kozawa, K. Okamoto, S. Tagawa, T. Kai, and T. Shimokawa, Jpn. J. Appl. Phys., 45 (2006) 6866.

17. K. Okamoto, R. Matsuda, H. Yamamoto, T. Kozawa, S. Tagawa, R. Fujiyoshi, and T. Sumiyoshi, Jpn. J. Appl. Phys., 52 (2013) 06GC04.

18. J. L. Lenhart, D. A. Fischer, S. Sambasivan, E. K. Lin, R. L. Jones, C. L. Soles, W. Wu, D. L. Goldfarb, and M. Angelopoulos, Langmuir, 21 (2005) 4007.

19. T. Hirayama, D. Shiono, S. Matsumaru, T. Ogata, H. Hada, J. Onodera, T. Arai, T. Sakamizu, A. Yamaguchi, H. Shiraishi, H. Fukuda, and M. Ueda, Jpn. J. Appl. Phys., 44 (2005) 5484

20. T. Hirayama, D. Shiono, J. Onodera, A. Yamaguchi, and H. Fukuda, Polym. Adv. Technol., 17 (2006) 116.

21. T. Fukuyama, T. Kozawa, S. Tagawa, R. Takasu, H. Yukawa, M. Sato, J. Onodera, I. Hirosawa, T. Koganesawa, and K. Horie, Appl. Phys. Express, 1 (2008) 065004.

22. T. Fukuyama, T. Kozawa, K. Okamoto, S. Tagawa, M. Irie, T. Mimura, T. Iwai, J. Onodera, I. Hirosawa, T. Koganesawa, and K. Horie, Jpn. J. Appl. Phys., 48 (2009) 06FC03.

23. T. Fukuyama, T. Kozawa, H. Yamamoto, S. Tagawa, M. Irie, T. Mimura, T. Iwai, J. Onodera, I. Hirosawa, T. Koganesawa, and K. Horie, J. Photopolym. Sci. Technol., 22 (2009) 105.

24. K. C. Tseng, N. J. Turro, and C. J. 
Durning, Phys. Rev., E 61 (2000) 1800.

25. D. S. Fryer, P. F. Nealey, and J. J. Pablo, J. Vac. Sci. Technol., B 18 (2000) 3376.

26. D. R. Medeiros, W. M. Moreau, K. Petrillo, M. Chauhan, W. S. Huang, C. Magg, D. Goldfarb, M. Angelopoulos, and P. Nealey, Proc. SPIE, 4345 (2001) 241.

27. L. Singh, P. J. Ludovice, and C. L. Henderson, Proc. SPIE, 5039 (2003) 1008 .

28. T. Kozawa, S. Tagawa, R. Ohnishi, T. Endo, and R. Sakamoto, Jpn. J. Appl. Phys., 50 (2011) 016504.

29. T. Kozawa and S. Tagawa, Jpn. J. Appl. Phys., 49 (2010) 030001.

30. T. Kozawa and S. Tagawa, Jpn. J. Appl. Phys., 50 (2011) 030209.

31. R. Hirose, T. Kozawa, S. Tagawa, T. Kai, and T. Shimokawa, Appl. Phys. Express, 1 (2008) 027004.

32. Y. Ikari, K. Okamoto, A. Konda, T. Kozawa, and T. Tamura, Jpn. J. Appl.
Phys., 59 (2020) 086506.

33. T. Kozawa, Jpn. J. Appl. Phys., 54 (2015) 056501 .

34. K. Natsuda, T. Kozawa, K. Okamoto, and S. Tagawa, Jpn. J. Appl. Phys., 45 (2006) L1256.

35. K. Natsuda, T. Kozawa, K. Okamoto, and S. Tagawa, Jpn. J. Appl. Phys., 46 (2007) 7285 .

36. T. Kozawa and S. Tagawa, Jpn. J. Appl. Phys., 50 (2011) 106502.

37. T. Kozawa, Jpn. J. Appl. Phys., 55 (2016) 056503.

38. G. P. Watson, D. Fu, S. D. Berger, D. Tennant, L. Fetter, A. Novembre, and C. Biddick, J. Vac. Sci. Technol., B 14 (1996) 4277.

39. H. Yamamoto, T. Kozawa, A. Nakano, K. Okamoto, Y. Yamamoto, T. Ando, M. Sato, H. Komano, and S. Tagawa, Jpn. J. Appl. Phys., 43 (2004) L848. 